\section{Epidemiologia de la malaria falciparum complicada: estudio de casos y controles en Tumaco y Turbo, Colombia, 2003}

\section{The epidemiology of complicated falciparum malaria: case and controls study in Tumaco and Turbo, Colombia, 2003}

\author{
Alberto Tobón C. \\ Carolina Giraldo S. \\ Juan Gabriel Pineros J. \\ Margarita Arboleda N. \\ Silvia Blair T. \\ Jaime Carmona-Fonseca \\ Grupo Malaria, Facultad de Medicina, Universidad de Antioquia.
}

Agradecimientos: La investigación fue posible gracias a las comunidades de Tumaco y Turbo, al personal de los hospitales locales "San Andrés" $y$ "Francisco Valderrama" y al apoyo de las entidades financiadoras. Destacamos el apoyo de la Dra. Sonia Gómez directora de la Secretaría de Salud de Nariño y del Dr. Cesar Arroyo del programa de control ETV. Al equipo profesional del Grupo Malaria de la Universidad de Antioquia que realizó los estudios de biología molecular y control de calidad de gota gruesa, especialmente agradecemos a las bacteriólogas Adriana Correa y Eliana Restrepo. A la Dra. María Patricia Arbeláez por la asesoría en el diseño del estudio y el análisis de datos.

Proyecto cofinanciado por Colciencias, Instituto Departamental de Salud de Nariño y Universidad de Antioquia.

Correspondencia: Sede de Investigación Universitaria. Calle 62 No. 52-59 Lab 610 - Medellín, Colombia.E-mail: malaria@quimbaya.udea.edu.co - albertobon@guajiros.udea.edu.co

\section{Resumen}

Objetivos: Identificar aspectos del hospedero, del parásito y del ambiente asociados con ocurrencia de malaria por Plasmodium falciparum complicada. Métodos: Estudio de casos y controles en pacientes de Tumaco y Turbo (Colombia) aplicando los criterios de complicación de la Organización Mundial de la Salud. Resultados: Entre noviembre 2002 y julio 2003 se captaron 64 casos (malaria complicada) y 135 controles (malaria no complicada). Las complicaciones fueron: hiperparasitemia $(40 \%)$, falla hepática $(36 \%)$, síndrome dificultad respiratoria aguda $(7 \%)$, falla renal $(4 \%)$, trombocitopenia grave $(3 \%)$, anemia grave (2\%), malaria cerebral $(2 \%)$ e hipoglicemia grave $(1 \%)$. Se encontraron como factores de riesgo para malaria falciparum complicada: a) Los antecedentes de malaria falciparum durante el último año fueron menores en los casos $(\mathrm{OR}=7.0(1.2-43.6)$ $\mathrm{P}=0.019)$; b) Mayor uso previo de antimaláricos en los casos (OR=2.2 (1.1-4.4) $\mathrm{P}=0.031)$ y c) mayor uso de cloroquina en los casos (OR=7.4 (1.1-7.8) $\mathrm{P}=0.017)$. Se hallaron los alelos MAD-20 y K1 del gen msp1 y FC-27 e IC-1 del gen msp2, cuya distribución de frecuencias fue similar entre casos y controles, aunque el alelo K1 mostró una variación importante entre grupos (casos: $9.4 \%$, controles: $3.5 \%$ ). La frecuencia de "signos de peligro" fue significativamente mayor en los casos $(\mathrm{OR}=3.3$, (1.5-7.4) $\mathrm{P}=0.001$ ). Los criterios de complicación malárica de la Organización Mundial de la Salud se comparan con otros y se discuten algunas implicaciones. Conclusión: Se identificaron como factores de riesgo para malaria falciparum complicada, la ausencia de antecedentes de malaria falciparum en el último año y el uso de antimaláricos antes de llegar al hospital.

Palabras clave: Malaria complicada, falciparum. Casos y controles. Colombia. 
Abstract

Objectives: Aimed at identifying host and parasite aspects associated to the presence of Plasmodium falciparum complicated malaria. Methods: Case and controls study in patients from Tumaco and Turbo (Colombia). We used the World Health Organization criteria to assess the presence of complicated malaria. Results: A total 64 cases and 135 controls were included between November 2002 and July 2003. Observed complications were hyperparasitaemia (40\%), liver failure (36\%), adult respiratory distress syndrome $(7 \%)$, renal failure $(4 \%)$, severe thrombocytopenia (3\%), severe anemia (2\%), cerebral malaria $(2 \%)$ and severe hypoglicemia (1\%). Were identified as risk factors: a) falciparum malaria history in the previous year was lower in the cases $(\mathrm{OR}=7.0(1.2-43.6)$ $\mathrm{P}=0.019)$, $\mathrm{b})$ the high use by the cases of antimalarials (OR=2.2, (1.1-4.4) $\mathrm{P}=0.031$ ) and c) the high use of chloroquine by the cases (OR=7.4 (1.1-7.8), $\mathrm{P}=0.017$ ) before attending to the hospital. Presence of $P$. falciparum alleles MAD-20 and K1 (mspl gene), FC-27 and IC-1 (msp2 gene) was confirmed. No significant differences were observed in the presence of these alleles; however K1 was more frequent in cases $(9.4 \%)$ than in controls $(3.5 \%)$. The frequency of danger signs during the disease was significantly greater in the cases $(\mathrm{OR}=$ 3.3 (1.5-7.4) $\mathrm{P}=0.001$ ). The World Health Organization criteria for complicated malaria are compared with others and some implications are discussed. Conclusion: They were identified as risk factors for complicated falciparum malaria, the absence of falciparum malaria antecedents in the last year and the use of antimalarials before attending to the hospital.

Keywords: Complicated malaria. Severe malaria, falciparum. Case and controls. Colombia.

\section{Introducción}

De las especies de Plasmodium que causan infección natural en el humano, $P$. falciparum ocupa el primer lugar en el mundo, tanto en la frecuencia de casos como en el número de complicaciones y muertes $^{1}$. No es completamente conocido cuál es el conjunto de causas (factores de riesgo) que hacen que unos pacientes con malaria falciparum presenten complicación de su enfermedad y otros no lo hagan. En la malaria, como en las demás infecciones, hay factores de riesgo -FR- que se consideran más asociados o dependientes del parásito, otros del hospedero humano y otros del entorno en que sucede la relación hospedero-parásito. Entre los FR más relacionados con el parásito se incluyen la especie, sus variaciones genéticas y la parasitemia ${ }^{2,3,4}$ los más relacionados con el hospedero se refieren al sexo y la edad $^{4,5,6}$, el estado nutricional y la inmuni$\operatorname{dad}^{7,8,9}$, el oficio, los antecedentes mórbidos y las conductas y comportamientos ${ }^{10,11}$ mientras los asociados al ambiente natural y social incluyen la zona de residencia, el clima, la fauna vectorial, el sistema de atención sanitaria y los patrones de producción y consumo de bienes y servicios, entre muchos otros ${ }^{10,12,13,14}$. Es la interacción entre estos factores y las asociaciones causales que establecen lo que, se supone, define la aparición de la enfermedad y la gravedad de la malaria.

Algunos clones de $P$. falciparum se han asociado con la presentación de formas clínicas graves y las infecciones policlonales podrían causar mayor parasitemia ${ }^{2,3}$. La hiperparasitemia se considera un factor de riesgo de complicación y el umbral para definirla puede variar según las condiciones de endemicidad de cada región ${ }^{15}$, por ejemplo en Colombia se han informado casos complicados con parasitemias menores de 50,000 parásitos/ $\mathrm{mL}^{16,17}$.

Las complicaciones de la malaria pueden ocurrir en cualquier edad; en algunas regiones de África la malaria complicada - 
MC- se presenta principalmente en niños menores de 5 años y en otras regiones del mundo ocurre tanto en niños como en adultos $4,6,7,18$.

La presentación clínica de la malaria depende mucho del estado de inmunidad y en pacientes con MC los niveles de inmunoglobulinas se correlacionan positivamente con la supervivencia; aunque se considera que la exposición repetida a la malaria limita la enfermedad grave por el desarrollo de inmunidad, esta no asegura la ausencia de complicación clínica, tal como se ha visto en poblaciones altamente expuestas a la malaria donde las complicaciones son frecuentes ${ }^{7,9}$.

La desnutrición induce deficiencia inmunitaria y aumenta el riesgo de desarrollar malaria, y se ha encontrado que reduce el riesgo de presentar malaria cerebral $^{19,20}$. Las comunidades afectadas por la desnutrición crónica usualmente padecen de parasitosis intestinales, en particular helmintiasis, y se ha informado que estas últimas se asocian con reducción del riesgo de malaria cerebral ${ }^{21}$, aunque también se ha informado su asociación con malaria complicada ${ }^{22}$.

El tiempo de evolución de la enfermedad antes de recibir un tratamiento apropiado se ha relacionado con la aparición de MC y es probable que su incidencia sea considerablemente mayor en comunidades que no tienen acceso rápido a la atención médica apropiada ${ }^{4,23}$. En Gambia se encontró asociación entre la duración de los síntomas en el momento de ingreso al hospital y el cuadro complicado; los niños con MC presentaron una duración media de los síntomas de 4 días, frente a 3 días en niños con malaria leve ${ }^{24}$.

La mayoría de los estudios sobre MC se han realizado en países de endemicidad palúdica alta, especialmente en países africanos. En América Latina los trabajos publicados sobre este tema son escasos; se han realizado pocos estudios sobre los FR para desarrollar malaria ${ }^{25,26,27}$ en los que se encontró que los antecedentes de malaria durante el último año, la no utilización de mosquiteros y las viviendas abiertas sin sistema de disposición de excretas fuera de ellas, son factores asociados con malaria. Otros trabajos ${ }^{28,29,30}$ describen casos complicados y su frecuencia, y un trabajo reciente informa la asociación de una secuencia específica del genoma de $P$. falciparum con $\mathrm{MC}^{31}$.

En Colombia se diagnosticaron 107,866 casos de malaria y 28 muertes en $2005^{32}$. En 2003, año de ejecución de este estudio, se diagnosticaron 164,722 casos, el $42 \%$ causados por $P$. falciparum, y se registraron 24 muertes $(21 \%$ de las muertes por malaria en América) ${ }^{1}$. Entre los años 2000 y 2004 se registraron 189 muertes en todo el país ${ }^{33}$, cifra que sólo da cuenta de los datos hospitalarios y está afectada por el subregistro, estimándose que ocurren anualmente 80 muertes $^{34}$.

En este artículo se da cuenta de los resultados de una investigación adelantada para identificar FR asociados a malaria falciparum complicada-MFC- en dos municipios de las regiones que más casos de malaria aportan en Colombia, la región del Pacífico (33\% de los casos) y la región de Urabá-Bajo Cauca (47\% de los casos) ${ }^{32}$.

\section{Materiales y métodos}

La población de referencia la constituyeron los pacientes con malaria por $P$. falciparum residentes en los municipios de Tumaco (costa del Pacifico) y Turbo (costa Atlántica) (Figura 1), de cualquier edad y sexo, residentes en zonas rural o urbana, de cualquiera de las etnias (negros, indígenas, mestizos). Se aplicó un diseño de casos y controles no pareados y se usaron sendas muestras representativas de las poblaciones de casos y controles, captados en forma secuencial en los hospitales locales de segundo nivel de ambos municipios entre noviembre de 2002 y julio de 2003. Los pacientes llegaron allí por sus propios medios o remitidos del puesto de diagnóstico de malaria y fueron captados según el orden de llegada, siempre y cuando cumplieran los criterios de inclusión 


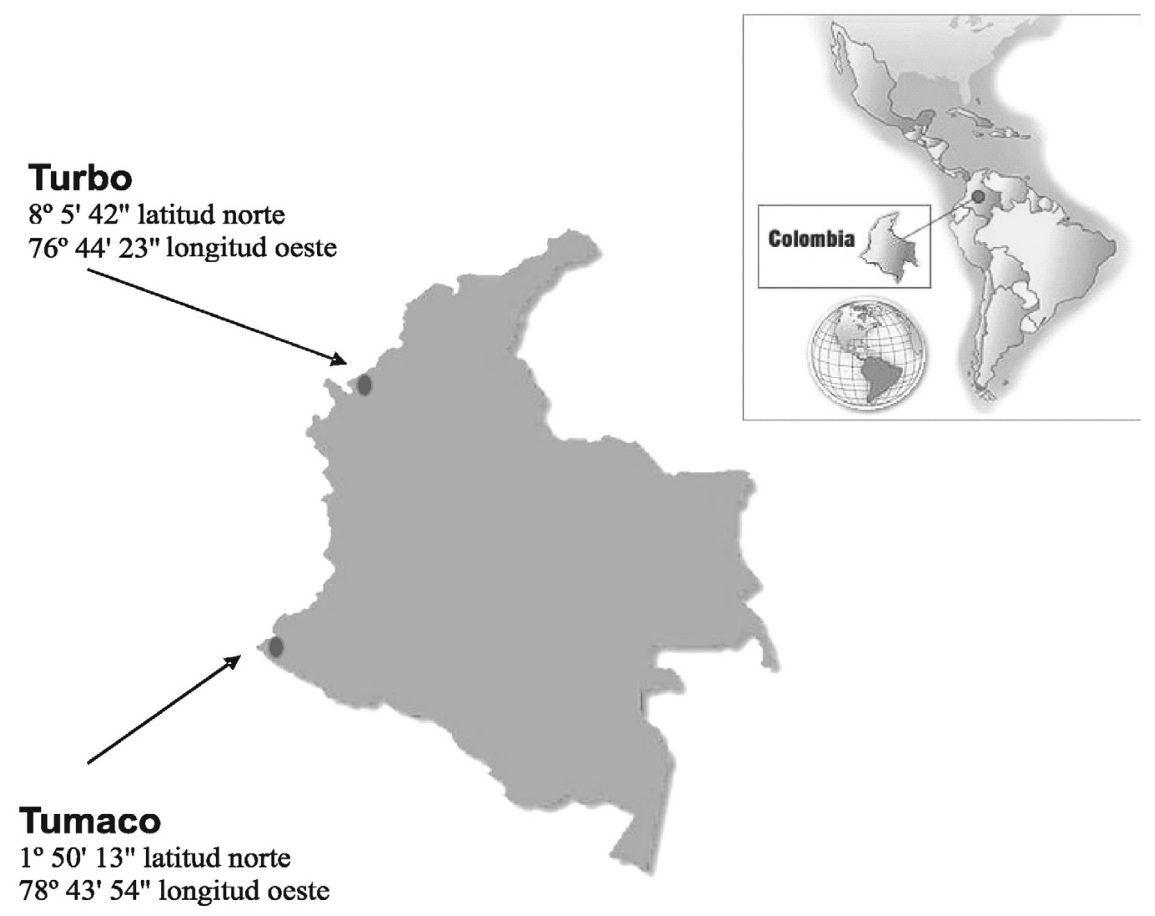

Figura 1 - Ubicación geográfica de Tumaco y Turbo, Colombia. Figure 1 - Geographic localization of Tumaco y Turbo, Colombia.

(tener diagnóstico de malaria solo por $P$. falciparum y dar el consentimiento) sin que ninguna otra condición interviniera. El proyecto se aprobó por el comité de ética de la Facultad de Medicina de la Universidad de Antioquia y de todos los pacientes se obtuvo el consentimiento informado y firmado.

Se definió como caso el paciente con malaria falciparum, diagnosticado por gota gruesa, y con criterios de complicación basados en los criterios definidos por OMS en $2000^{15}$, excepto que la hiperparasitemia se definió con la pauta establecida en Colombia ${ }^{34} \mathrm{y}$ la trombocitopenia grave que no está definida por OMS, y que nosotros definimos como un recuento de menos de 20,000 plaquetas por microlitro de sangre de acuerdo al criterio de Horstmann et al. (1981) mencionado en la guía OMS ${ }^{15}$. Las complicaciones de malaria falciparum estudiadas se definen en la Tabla 1. Se definió como control al paciente con malaria falciparum sin complicaciones. Esta clasificación fue realizada por médicos me- diante examen clínico y de laboratorio y además se evaluó la presencia de signos de peligro ${ }^{35}$ al momento de la consulta. Se hizo seguimiento para asegurar la cura clínica y parasitológica a todos los pacientes, hasta el alta hospitalaria (casos) o en forma ambulatoria; un control se tornó en caso durante el seguimiento.

El diagnóstico de $P$. falciparum se realizó mediante lectura de gota gruesa elaborada según lo definido por la OMS y se calculó la parasitemia por microlitro de sangre con base en el número de parásitos por 100 leucocitos (considerando una media de 8,000 leucocitos $\left./ \mathrm{mm}^{3}\right)^{36}$. Las mediciones de laboratorio clínico se efectuaron en los hospitales locales con equipos automatizados (analizador hematológico MEK-8918 y espectrofotómetro RA-50). Se genotipificaron los plasmodios mediante la prueba de reacción en cadena de la polimerasa ${ }^{37}$.

En un formulario precodificado se captó información sobre antecedentes, condiciones socioeconómicas, prácticas de salud, zona de residencia, y se hicieron 
preguntas para valorar la accesibilidad a la atención en salud.

El número de casos se definió según estos criterios: nivel de confianza 95\%, potencia de la prueba $80 \%$, relación entre los tamaños de los grupos "casos" y "controles” $1: 2$, frecuencia esperada de exposición al factor de riesgo en los "controles" $15 \%$, valor mínimo de la razón de apuestas (conocida en la literatura inglesa como odds ratio, OR) para ser detectada 3.0; se calcularon muestras de 62 casos y 124 controles.

La información obtenida se procesó y analizó con los programas EpiInfo 6.04 y SPSS 10.0. Se hizo análisis de normalidad de cada variable mediante la prueba de Kolmogorov-Smirnov. Se ejecutó un análisis de casos y controles no pareados aplicando una prueba Chi cuadrada según Mantel y Haenszel; se calculó el OR para medir la magnitud de la diferencia de riesgo entre casos y controles; y se obtuvo el intervalo de confianza del 95\% (IC95\%) para cada OR. En todos los análisis se consideró estadísticamente significativa una probabilidad menor de 0.05 (5\%).

\section{Resultados}

Se ingresaron al estudio 199 pacientes con malaria falciparum: 64 casos (Tabla 1) y 135 controles; se diagnosticó simultáneamente más de una complicación en 10 pacientes, siendo encontradas 77 complicaciones en total (Tabla 2). Ninguno de los pacientes murió.

El tiempo de evolución de la malaria actual hasta ingresar al estudio osciló entre 1 a 30 días, con promedio de 5.6 días (Me: 5, D.E. 3.0) en los casos y de 5.9 (Me: 4; D.E. 4.4) en los controles (Tabla 3). La

Tabla 1 - Criterios empleados para el diagnóstico de malaria complicada (casos).

Table 1 - Criteria used for the diagnosis of complicated malaria (cases).

\begin{tabular}{|c|c|}
\hline Diagnóstico y criterio de complicación & $\operatorname{casos}(n=64)$ \\
\hline UNA COMPLICACION $(n=54)$ & Frec. (\%) \\
\hline \multicolumn{2}{|l|}{ Hiperparasitemia: } \\
\hline$>50,000$ parásitos asexuales $/ \mu L^{*}$ & $25(39.0)$ \\
\hline \multicolumn{2}{|l|}{ Falla hepática: } \\
\hline Bilirrubina total BT $>3.0 \mathrm{mg} / \mathrm{dL}$ o aspartato-aminotransferasa AST > 140 U.I. & $21(32.7)$ \\
\hline \multicolumn{2}{|l|}{ Síndrome de dificultad respiratoria aguda S.D.R.A.: } \\
\hline Disnea con hallazgos radiológicos de edema pulmonar o infiltrado pulmonar. & $3(4.6)$ \\
\hline \multicolumn{2}{|l|}{ Falla renal: } \\
\hline Creatinina $>3.0 \mathrm{mg} / \mathrm{dL}$ ó nitrógeno uréico en sangre BUN >40 mg\% & $1(1.6)$ \\
\hline \multicolumn{2}{|l|}{ Trombocitopenia grave: } \\
\hline Plaquetas $<20,000 / \mu L^{*}$ & $1(1.6)$ \\
\hline \multicolumn{2}{|l|}{ Anemia grave: } \\
\hline Hemoglobina $<5.0 \mathrm{mg} / \mathrm{dL}$ & $1(1.6)$ \\
\hline \multicolumn{2}{|l|}{ Hipoglicemia grave: } \\
\hline Glicemia $<40 \mathrm{mg} / \mathrm{dL}$ & $1(1.6)$ \\
\hline \multicolumn{2}{|l|}{ Malaria cerebral: } \\
\hline Coma según escala de coma Glasgow $<9$ ó Blantyre $<2$ & $1(1.6)$ \\
\hline \multicolumn{2}{|l|}{ MAS DE UNA COMPLICACION $(n=10)$} \\
\hline Falla hepática + hiperparasitemia. & $3(4.6)$ \\
\hline Falla hepática + trombocitopenia. & $2(3.1)$ \\
\hline Falla hepática + S.D.R.A. & $1(1.6)$ \\
\hline Falla renal + hiperparasitemia. & $1(1.6)$ \\
\hline S.D.R.A. + hiperparasitemia. & $1(1.6)$ \\
\hline Falla renal + malaria cerebral + hiperparastitemia. & $1(1.6)$ \\
\hline Falla renal + falla hepática + anemia grave + S.D.R.A. & $1(1.6)$ \\
\hline
\end{tabular}

* Criterios adaptados por los investigadores. Criteria adapted by the researchers. 
Tabla 2 - Frecuencia de las complicaciones por municipio.

Table 2 - Frequency of complications by municipality.

\begin{tabular}{lccc}
\hline Complicaciones * & $\begin{array}{c}\text { Tumaco } \mathrm{n}=37 \\
\text { Frec. }(\%)\end{array}$ & $\begin{array}{c}\text { Turbo } \mathrm{n}=40 \\
\text { Frec. }(\%)\end{array}$ & $\begin{array}{c}\text { Total } \mathrm{n}=77 \\
\text { Frec. }(\%)\end{array}$ \\
\hline Hiperparasitemia & $18(48)$ & $13(33)$ & $31(40)$ \\
Falla hepática & $11(30)$ & $17(43)$ & $28(36)$ \\
S.D.R.A. & $2(5)$ & $4(10)$ & $6(7)$ \\
Falla renal & $3(8)$ & $1(2)$ & $4(5)$ \\
Trombocitopenia grave & $1(3)$ & $2(5)$ & $3(4)$ \\
Anemia grave & $0(0)$ & $2(5)$ & $2(3)$ \\
Malaria cerebral & $1(3)$ & $1(2)$ & $2(3)$ \\
Hipoglicemia grave & $1(3)$ & $0(0)$ & $1(2)$ \\
* 77 complicaciones que se diagnosticaron en 64 pacientes (ver tabla 1). 77 complications that were diagnosed in 64 patients (see table 1).
\end{tabular}

Tabla 3 - Duración de la enfermedad hasta el ingreso y frecuencia de síntomas y signos.

Table 3 - Disease duration before admission and frequency of symptoms and signs.

\begin{tabular}{|c|c|c|c|c|c|}
\hline Días de evolución de enfermedad * & $\begin{array}{c}\text { Casos } \\
\mathrm{n}=64(\%)\end{array}$ & $\begin{array}{l}\text { Controles } \\
n=135 \text { (\%) }\end{array}$ & $\begin{array}{c}\text { Total } \\
\mathrm{n}=199(\%)\end{array}$ & & \\
\hline 1 & $1(2)$ & $3(2)$ & $4(2)$ & & \\
\hline 2 & $5(8)$ & $15(11)$ & $20(10)$ & & \\
\hline 3 & $14(22)$ & $24(18)$ & $38(19)$ & & \\
\hline $4-14$ & $42(65)$ & $82(61)$ & $126(63)$ & & \\
\hline $15-30$ & $2(3)$ & $11(8)$ & $136)$ & & \\
\hline Síntomas o signos durante la enfermedad & $\%$ & $\%$ & $\%$ & OR (IC) ${ }^{1}$ & $\mathbf{P}^{2}$ \\
\hline Fiebre & $63(98)$ & $132(98)$ & $195(98)$ & & N.S. ${ }^{3}$ \\
\hline Escalofrío & $57(89)$ & $126(93)$ & $183(92)$ & & N.S. \\
\hline Dolor de cabeza & $57(89)$ & $123(91)$ & $180(90)$ & & N.S. \\
\hline Sudoración & $59(92)$ & $119(88)$ & $178(89)$ & & N.S. \\
\hline Dolor osteomuscular & $53(83)$ & $111(82)$ & $164(82)$ & & N.S. \\
\hline Dolor abdominal & $42(66)$ & $86(64)$ & $129(65)$ & & N.S. \\
\hline Vomito & $31(48)$ & $66(49)$ & $97(49)$ & & N.S. \\
\hline Tos & $26(41)$ & $57(42)$ & $83(42)$ & & N.S. \\
\hline Diarrea & $18(28)$ & $32(24)$ & $50(25)$ & & N.S. \\
\hline Dificultad respiratoria & $24(38)$ & $19(14)$ & $43(22)$ & $3.7(1.7-7.8)$ & 0.00018 \\
\hline \multicolumn{6}{|l|}{ Signos al exámen clínico } \\
\hline Ictericia & $21(33)$ & $13(10)$ & & $4.6(2.1-9.9)$ & 0.00005 \\
\hline Frecuencia respiratoria alta & $31(48)$ & $37(27)$ & & $2.5(1.3-4.6)$ & 0.004 \\
\hline Hiperpirexia ( $\mathrm{t}^{\circ}$ axilar $>39.5^{\circ} \mathrm{C}$ ) & $12(19)$ & $10(7)$ & & $2.9(1.1-7.8)$ & 0.017 \\
\hline Trastornos neurologicos & $5(8)$ & $2(1)$ & & $5.6(1.1-29.8)$ & 0.042 \\
\hline
\end{tabular}

1 OR: odds ratio, IC: intervalo de confianza (confidence interval).

$2 \mathrm{P}$ : valor de significancia; en negrita $\mathrm{P}<0.05$ ( $\mathrm{P}$ value, in bold $\mathrm{P}$ value $<0.05$ )

${ }^{3}$ N.S. No significativo. Non significant.

*Chi²: 2.79, P=0,597 
frecuencia de síntomas que se presentaron durante la enfermedad fue similar en ambos grupos; excepto la dificultad respiratoria y la ictericia que fueron más frecuentes en los casos que en los controles (Tabla 3). En el momento de consultar se encontró mayor frecuencia en los casos que en los controles de ictericia, taquipnea, hiperpirexia $\left(\mathrm{T}^{\mathrm{o}}\right.$ axilar $>39.5^{\circ} \mathrm{C}$ ) y alteración neurológica (somnolencia, confusión, irritabilidad) en los casos que en los controles $(P<0.05)$ (Tabla 3$)$.

\section{Evaluación de los factores de riesgo en casos y controles (Tabla 4)}

Características sociodemográficas de los pacientes. La edad de los pacientes osciló entre 1 y 82 años (promedio 26 años, mediana 23); El 71\% de los pacientes residía en zona rural. Los casos tenían un promedio de permanencia en zonas endémicas de malaria de 16 años y los controles una media de 20 años. No se encontró diferencia estadísticamente significativa (D.E.S.) entre casos y controles según estas características demográficas.

El 73\% de los pacientes o sus familias tuvo ingresos mensuales inferiores al salario mínimo legal vigente (aproximadamente U.S. \$110) y solo $4 \%$ tuvo salarios superiores a dos veces el salario mínimo legal. El promedio de dinero disponible para consultar fue U.S. $\$ 6$ y $58 \%$ de los pacientes disponía de menos de U.S. \$1,5 para consultar. No se encontraron diferencias significativas entre casos y controles para estas variables.

Antecedentes de malaria, infección y embarazo. 55\% de los casos y $49 \%$ de los controles no presentaron antecedentes de malaria; no se encontró D.E.S. entre casos y controles según el número de antecedentes palúdicos en la vida o por especie $(P$. vivax o $P$. falciparum). Los pacientes con antecedente de malaria en el último año solo por $P$. vivax presentaron mayor riesgo de tener $\mathrm{MFC}$ respecto a quienes no lo tuvieron $(\mathrm{OR}=4.0(1.0-17.3)$ Fisher=0.040) y entre quienes tuvieron algún antecedente de malaria, fue más frecuente malaria falciparum en los controles (OR=7.0 (1.243.6) Fisher=0.019). Otros antecedentes encontrados fueron enfermedad infecciosa concomitante y embarazo, con frecuencia similar en ambos grupos $(P>0.05)$.

Conductas y prácticas en salud. Entre quienes habían consultado previamente durante el episodio actual de la enfermedad a otro agente de salud, la media aritmética del tiempo transcurrido desde el inicio de la enfermedad hasta consultar fue de 4.1 días en los casos ( $\mathrm{Me}=3$, D.E. 2.8) y 4.5 días en los controles $(\mathrm{Me}=4$, D.E. 3.2); el $30 \%$ demoraron al menos 7 días para consultar. Entre ellos, a 32\% (25/79) les recetaron antimaláricos y les enseñaron cómo tomarlos, pero la proporción de quienes no aprendieron a hacerlo fue mayor en los casos $(7 / 11,64 \%)$ que en los controles $(4 / 14,29 \%)$.

De 41 pacientes que habían tomado previamente algún antimalárico $(\mathrm{OR}=2.2$, (1.1-4.4) $\mathrm{P}=0.031), 16$ (39\%) se automedicaron, 8 (13\%) casos y 8 (6\%) controles. La cloroquina como monoterapia fue más usada en los casos, $\mathrm{OR}=7.37$ (1.1-7.8) $P$ $=0.017$ ) y solo un paciente recibió el medicamento adecuado para tratar la malaria falciparum en Colombia. El acetaminofén - sólo o combinado con otro fármacofue el medicamento más usado durante la enfermedad: $67 \%$ de los casos y $68 \%$ de los controles. El 25\% (50) de los enfermos tomaron preparados con plantas (casos 22\%, controles 27\%) (P> 0.05).

Condiciones de acceso a la atención en salud. El 63\% de los casos y $64 \%$ de los controles tenían afiliación al sistema de seguridad social en salud ( $P>0.05$ ). La duración de los diferentes momentos de la atención, tomados individualmente o sumados, no presentó D.E.S. entre los casos y los controles.

Genotipos de los parásitos. Se encontraron las familias alélicas MAD20, K1 y 
Tabla 4 - Estimación del riesgo para malaria complicada según características sociodemográficas y antecedentes, prácticas y acceso a la atención en salud.

Table 4 - Estimation of risk for complicated malaria according to sociodemographic characteristics, health antecedents and practices, and access to health care.

\begin{tabular}{|c|c|c|c|c|c|c|}
\hline Variable & Categorías & $\begin{array}{c}\text { Casos } \\
\mathrm{n}\end{array}$ & $\begin{array}{c}\text { Controles } \\
n\end{array}$ & $\mathrm{OR}^{1}$ & $I C^{2}$ & $\mathrm{P}^{3}$ \\
\hline \multicolumn{7}{|l|}{ Características socio-demográficas } \\
\hline \multirow[t]{2}{*}{ Sexo } & masculino & 34 & 71 & 0.98 & $0.54-1.77$ & 0.944 \\
\hline & femenino & 30 & 64 & & & \\
\hline \multirow[t]{2}{*}{ Edad } & $<15$ & 21 & 36 & 1.34 & $0.67-2.69$ & 0.372 \\
\hline & $\geq 15$ & 43 & 99 & & & \\
\hline \multirow[t]{2}{*}{ Zona de procedencia } & urbana & 14 & 18 & 1.82 & $0,840-3.94$ & 0.129 \\
\hline & rural & 50 & 117 & & & \\
\hline \multirow[t]{2}{*}{ Estadía últimos 15 días } & urbana & 16 & 21 & 1.81 & $0,82-4.0$ & 0.111 \\
\hline & rural & 48 & 114 & & & \\
\hline \multirow[t]{2}{*}{ Permanencia en zona malárica } & $<10$ años & 25 & 36 & 1.76 & $0,94-3.33$ & 0.078 \\
\hline & $>10$ años & 39 & 99 & & & \\
\hline \multicolumn{7}{|c|}{ Antecedentes de malaria, infección y embarazo } \\
\hline \multirow[t]{2}{*}{ Antecedente malaria ultimo año** } & P. vivax & 7 & 4 & 7.0 & $1.24-43.6$ & $0.019 *$ \\
\hline & P. falciparum & 6 & 24 & & & \\
\hline \multirow[t]{2}{*}{ Antecedente ultimo año $P$. vivax } & $\mathrm{Si}$ & 7 & 4 & 4.0 & $1.02-17.3$ & $0.040 *$ \\
\hline & No & 57 & 131 & & & \\
\hline \multirow[t]{2}{*}{ Embarazo** } & $\mathrm{Si}$ & 8 & 16 & 1.09 & $0.36-3.24$ & 0.863 \\
\hline & No & 22 & 48 & & & \\
\hline \multirow[t]{2}{*}{ Infección concomitante } & $\mathrm{Si}$ & 16 & 28 & 1.27 & $0.63-2.57$ & 0.499 \\
\hline & No & 48 & 107 & & & \\
\hline \multicolumn{7}{|l|}{ Conductas y prácticas en salud } \\
\hline \multirow[t]{2}{*}{ Uso previo de antimaláricos } & $\mathrm{Si}$ & 19 & 22 & 2.17 & $1.07-4.39$ & 0.031 \\
\hline & No & 45 & 113 & & & \\
\hline \multirow[t]{2}{*}{ Uso previo de cloroquina $\mathrm{CQ}$} & $\mathrm{Si}$ & 12 & 10 & 7.37 & $1.08-7.76$ & 0.017 \\
\hline & No & 52 & 125 & & & \\
\hline \multirow[t]{2}{*}{ Uso previo $\mathrm{CQ}+$ otro antimalárico } & $\mathrm{Si}$ & 17 & 19 & 2.21 & $0.99-4.91$ & 0.03 \\
\hline & No & 47 & 116 & & & \\
\hline \multirow[t]{2}{*}{ Automedicación antimalárica } & $\mathrm{Si}$ & 8 & 8 & 2.27 & $0.73-7.07$ & 0.112 \\
\hline & No & 56 & 127 & & & \\
\hline \multirow[t]{2}{*}{ Uso previo drogas No antimaláricas** } & $\mathrm{Si}$ & 52 & 103 & 1.33 & $0,62-2.88$ & 0.467 \\
\hline & No & 11 & 29 & & & \\
\hline \multirow[t]{2}{*}{ Uso de plantas } & $\mathrm{Si}$ & 14 & 36 & 0.77 & $0.36-1.64$ & 0.467 \\
\hline & No & 50 & 99 & & & \\
\hline \multicolumn{7}{|l|}{ Condiciones de acceso a la atención en salud } \\
\hline \multirow[t]{2}{*}{ Consultó antes (extrahospital 1a. Consulta) } & no & 36 & 84 & 0.78 & $0,43-1.43$ & 0.422 \\
\hline & si & 28 & 51 & & & \\
\hline \multirow[t]{2}{*}{ Aprendió a tomar antimalárico** } & no & 7 & 4 & 4.38 & $0.62-35.2$ & 0.088 \\
\hline & si & 4 & 10 & & & \\
\hline \multirow[t]{2}{*}{ Costo del transporte (U.S.\$) } & $0-1.5$ & 26 & 43 & 1.46 & $0.75-2.84$ & 0.225 \\
\hline & $>1.5$ & 38 & 92 & & & \\
\hline Costo de la atención (U.S.\$)** & $0-1.5$ & 35 & 102 & 0.46 & $0.19-0.84$ & 0.007 \\
\hline & $>1.5$ & 23 & 27 & & & \\
\hline Costo del medicamento (U.S.\$) & $0-1.5$ & 55 & 128 & 1.43 & $0.15-1.41$ & $0.089^{*}$ \\
\hline & $>1.5$ & 3 & 1 & & & \\
\hline Disponible consulta salud (U.S.\$/mes)** & $0-150$ & 32 & 77 & 0.75 & $0.4-1.43$ & 0.352 \\
\hline & $>150$ & 32 & 58 & & & \\
\hline
\end{tabular}

'OR: odds ratio,

${ }^{2}$ IC: intervalo de confianza (confidence interval),

${ }^{3} \mathrm{P}$ : valor de significancia; en negrita $\mathrm{P}<0.05$ ( $P$ value, in bold $\mathrm{P}$ value $<0.05$ )

*Fisher 1 cola (Fisher, 2 tailed).

**Valor de $\mathrm{n}$ menor al total de casos o controles ( $\mathrm{n}$ value lower than total of cases and controls) 
RO33 (gen mspl, proteína 1 de Superficie del merozoíto), FC27 e IC1 (gen msp2, proteína 2 de superficie del merozoíto) y glurp 500, 600, 650, 700, 800, 900 y 1000 pb (gen glurp, proteína rica en glutamato). La frecuencia en los casos y en los controles, respectivamente para cada alelo fue: MAD-20, $98 \%$ y $99 \%$; K1-200, 9\% y 4\%; RO-33, $2 \%$ y 2\%; IC1-500, 64\% у 51\%; FC27-300, 20\% y 16\%; GLURP-650, 20\% y 16\%; GLURP-700, 25 y $24 \%$; GLURP-800, 15\% y 15\% (P>0.05).

\section{Discusión}

De acuerdo con las características sociodemográficas descritas para los pacientes de este estudio no hubo diferencias importantes entre casos y controles respecto a su distribución por sexo, edad, zona de residencia, procedencia o tiempo de permanencia en zonas maláricas.

Los síntomas que presentaron los casos durante la enfermedad son similares a aquellos presentes en los controles. La frecuencia de presentación de los síntomas clásicos de la malaria en los casos es alta, comparada con la informada en 291 pacientes con malaria grave estudiados en Colombia ${ }^{16}$, quienes presentaron fiebre (95\%), escalofrío (49\%) y sudoración (37\%); la frecuencia de vómito y diarrea fue similar en ambos estudios: $58 \%$ y $27 \%$, respectivamente, en los pacientes del estudio previo, frente a $52 \%$ y $26 \%$ en esta investigación. De otro lado, la frecuencia de signos y síntomas durante la enfermedad, tanto en casos como en controles, es similar a lo informado en pacientes con malaria vivax no complicados en Colombia ${ }^{38}$ en quienes los principales hallazgos fueron fiebre $(99 \%)$, cefalea $(99 \%)$, escalofrío (91\%), sudoración (78\%) y vómito (39\%). De acuerdo con lo anterior, los signos y síntomas clásicos presentes durante la enfermedad no permiten distinguir entre malaria complicada y no complicada, y tampoco son útiles para diferenciar la especie de plasmodio causante de la infección. Sin embargo, otros signos y síntomas son considerados signos de peligro ${ }^{35}$ y se encuen- tran con mayor frecuencia en pacientes complicados; en este estudio encontramos mayor frecuencia en los casos que en los controles de dificultad respiratoria, ictericia, hiperpirexia y alteración neurológica.

El problema de los criterios diagnósticos de MC. El diagnóstico de MFC se estableció en este estudio con los criterios establecidos por la OMS en $2000^{15}$, excepto para la hiperparasitemia, en la cual usamos el criterio establecido en Colombia por el Ministerio de Salud ${ }^{34}$. El 84\% de los casos presentaron complicación única: Las complicaciones más frecuentes fueron hiperparasitemia (40\%) y falla hepática (36\%), y en el $16 \%(10 / 64)$ de los casos se presentó más de una complicación; la falla hepática fue la que más se acompañó de otras complicaciones (7/10).

En los países de endemicidad alta y estable, se considera que una parasitemia mayor de $4 \%$ de glóbulos rojos parasitados (aproximadamente 200,000 parásitos/ $\mu \mathrm{L}$ ) aumenta el riesgo de complicación y se acepta que cuando no se tienen cifras locales puede usarse como referencia un $20 \%$ de grp para definir $\mathrm{MC}^{15}$. En Colombia, lo usual es encontrar casos graves con menos de 100,000 parásitos $/ \mu L^{16,17,39}$, lo que llevó al Ministerio de Salud a fijar el punto de corte en 50,000 parásitos $/ \mu L$. Entre nuestros 64 casos, 31 presentaron más de 50,000 parásitos/mL, 25 de los cuales carecían de criterios clínicos de complicación (8 de estos con mas de 100,000 parásitos/mL). Más importante aún es que el $52 \%$ de los casos fue clasificado así por criterios clínicos, aunque no tenían más de 50,000 parásitos/mL (media 15.209) y sólo $10 \%$ de los casos presentó hiperparasitemia, simultáneamente con uno o varios criterios clínicos. La hiperparasitemia no es considerada en sí misma por algunos investigadores como un signo de gravedad, si no está acompañada de una disfunción orgánica ${ }^{4}$. De otro lado, se considera que la ausencia de una densidad parasitaria alta no asegura la ausencia de complicación, lo que puede ocurrir espe- 
cialmente en individuos no inmunes ${ }^{15}$, tal como sucedió con el $52 \%$ de nuestros casos. Estos hallazgos hacen pensar que para las zonas estudiadas debe mantenerse el criterio recomendado por las autoridades sanitarias colombianas.

Otro criterio diagnóstico de MFC de la OMS es la glicemia inferior a $40 \mathrm{mg} / \mathrm{dL}$. Esta variable presentó una media similar en casos y controles (106 vs. $105 \mathrm{mg} / \mathrm{dL}$ ) y solo encontramos un paciente con glicemia $<40 \mathrm{mg} / \mathrm{dL}$, mientras que con el criterio fisiológico, aplicado por el laboratorio donde se hizo la medición (<70 mg/ dL), 5 casos presentaron hipoglicemia, es decir que el criterio OMS dejó sin detectar 4 casos. Estos 4 pacientes tenían de 14 a 57 años de edad, son 1 hombre y 3 mujeres (gestantes), 3 tienen hemoglobina igual o menor que $9 \mathrm{~g} / \mathrm{dL}$ y la parasitemia oscila entre 40800 y 134800 parásitos/mL. El criterio de la OMS deja por fuera 4 pacientes en situación crítica, entre ellos 3 gestantes con anemia e hiperparasitemia.

Según el criterio OMS el diagnóstico de falla renal se hace con un valor de creatinina mayor de $3 \mathrm{mg} / \mathrm{dL}$ o de BUN ("blood ureic nitrogen”) mayor de $40 \mathrm{mg} / \mathrm{dL}$ en pacientes con oliguria o anuria, aunque se acepta que la diuresis puede estar normal o aumentada. En la práctica clínica se aceptan como límites de anormalidad (pero no de falla renal) 1.5 y $20 \mathrm{mg} / \mathrm{dL}^{40}$ respectivamente. Ninguno de los casos tuvo creatinina $>3$ mg/dL y 4 tuvieron BUN >40 mg/dL; por otra parte, 4 casos mostraron creatinina $>1.5 \mathrm{mg} / \mathrm{dL}$ y 15 casos presentaron BUN >20 $\mathrm{mg} / \mathrm{dL}$, de los cuales 2 presentaron elevadas ambas variables. Combinando la creatinina y el BUN, estos dos últimos pacientes tienen probablemente una falla renal, si se aplican los límites de Harrison pero no según el criterio OMS. Se trata de dos hombres de 12 y 42 años con fallas multisistémicas y un índice BUN/creatinina de 29.5 y 31.5, que superan ampliamente el valor de 20 considerado diagnóstico de falla renal aguda ${ }^{41}$.

El criterio OMS para falla hepática es de bilirrubina total $\mathrm{BT}>3.0 \mathrm{mg} / \mathrm{dL}$ o de enzima AST (aspartato aminotransferasa) >120 UI. Entre los 64 casos, hubo 15 de falla hepática según la BT y 17 según la AST, mientras 4 pacientes presentaron ambas pruebas elevadas, de los cuales 3 tienen ictericia. El criterio de anormalidad más usado es $\mathrm{BT}>1.0 \mathrm{mg} / \mathrm{dL}$ y AST > $40 \mathrm{UI}^{40}$; entre los 64 casos, hay 38 con BT $>1.0 \mathrm{mg} /$ dL, 34 con AST > 40 UI y 25 con ambos valores elevados. De estos 25 casos con anormalidad simultánea en BT y AST, 13 tienen además, bilirrubina directa mayor de $0.3 \mathrm{mg} / \mathrm{dL}$ e ictericia. Si se aplica el criterio de OMS, solo 3 de estos 13 pacientes que reúnen 4 criterios de disfunción hepática, serían diagnosticados con falla hepática.

En consecuencia, los criterios de OMS parecen demasiado exigentes, por lo cual su sensibilidad es baja y por ello pareciera conveniente profundizar en el estudio de estos criterios en pacientes con malaria de zonas de endemicidad baja como es el caso colombiano.

\section{El papel de los supuestos factores de riesgo de la génesis de la MFC (Tabla 4).}

Los alelos encontrados de los genes msp1, msp2 y glurp presentan una frecuencia similar en casos y controles $(\mathrm{P}>0.05)$. El alelo $\mathrm{K} 1$ se ha asociado en distintas regiones del mundo con $\mathrm{MFC}^{2,42}$ pero en otros lugares no se encontró esta asociación ${ }^{43,44}$. Nosotros encontramos mayor frecuencia de $\mathrm{K} 1$ en los casos (6) que en los controles (5) aunque la frecuencia es baja (9.4\% vs. $3.7 \%, \mathrm{P}>0.05)$. El alelo RO33 que se ha asociado con mayores niveles de Factor de Necrosis Tumoral alfa y con mayor frecuencia en pacientes con $\mathrm{MFC}^{45}$, solo lo encontramos en $2 \%$ de casos y controles $(\mathrm{P}>0.05)$. En Colombia hay poca variabilidad genética de $P$. falciparum ${ }^{37}$, lo que se refleja en la similitud de aislados de nuestros casos y controles; de otro lado, hay diferencias importantes con otras regiones del mundo donde son más frecuentes otras familias alélicas, como K1 y RO33, que podrían explicar diferencias geográficas en la morbilidad complicada. 
Las preguntas sobre antecedentes de malaria apuntaban a explorar la relación entre la inmunidad antimalárica y el hecho de que el actual episodio palúdico se complicara o no. Haber padecido o no malaria en cualquier momento de la vida no mostró diferencia entre los dos grupos (OR=1.3 (0.7-2.5) $\mathrm{P}=0.389$ ), como tampoco lo hizo la misma variable considerada en términos del número de episodios padecidos. Entre quienes presentaron uno o más antecedentes de malaria el último año, malaria falciparum fue mas frecuente en los controles, lo que pudo disminuir la probabilidad de complicación posiblemente por la inmunidad que se obtiene por infecciones repetidas ${ }^{7}$. De manera contraria, entre quienes tuvieron antecedente de malaria y que tuvieron malaria vivax, fue mayor el riesgo de complicación actual (OR=4.0 (1.0-17.3) $\mathrm{P}=0.040)$.

Dos condiciones que se han asociado con mayor riesgo para MC son la gestación y la edad menor de 5 años ${ }^{4,6,18}$. Contrariamente, nosotros encontramos que fue similar en ambos grupos la proporción de niños menores de 5 años de edad (OR=0.69, (0.24-2.03), $\mathrm{P}=0.501)$ y la proporción de mujeres embarazadas (OR=1.09, (0.36-3.24), $\mathrm{P}=0.863)$.

Las conductas y prácticas en salud del individuo y su grupo familiar han sido señaladas como posibles factores de riesgo para $\mathrm{MC}^{12,14}$. Nosotros encontramos que $30 \%$ de los casos hizo uso previo de antimaláricos - automedicados o recetados- en el actual episodio, comparado con $16 \%$ de los controles (OR=2.2, (1.1-4.4) $\mathrm{P}=0.031$ ), lo cual sugiere que emplear estos medicamentos constituye un riesgo de complicación, quizás porque son mal usados, lo que es ratificado por nuestro hallazgo de que apenas $11 \%$ de los casos y $18 \%$ de los controles hizo uso "adecuado y oportuno” de los antimaláricos. El uso de estos antimaláricos por automedicación no mostró diferencia entre casos y controles ( $13 \%$ y $6 \%$, en su orden).

La demora por consultar es señalada como un factor de riesgo de $\mathrm{MC}^{23,24}$. Nues- tros datos indican que esta variable no contribuye a explicar la complicación, pues la demora entre el inicio de los síntomas y la consulta a cualquier agente de salud o al hospital donde se captaron los pacientes es similar en ambos grupos: 4.1 y 4.5 días la primera y 5.6 y 5.9 días la segunda. De 45 a $52 \%$ de los pacientes tuvo una demora hasta de 3 días para consultar a cualquier agente de salud, cifra que interpretamos como razonable, no pudiendo señalarse que exista demora para consultar en estos pacientes; solo $22 \%$ de los casos y $28 \%$ de los controles tardaron 6 o más días para hacer la consulta.

El tiempo transcurrido desde la llegada del paciente al hospital hasta el inicio del tratamiento fue mayor de 5 horas para $81 \%$ de los casos y $76 \%$ de los controles. En general, el tiempo requerido para obtener una atención sanitaria efectiva fue largo, puesto que una espera mayor de 5 horas para iniciar el tratamiento a un paciente con malaria, inclusive no complicada, parece inaceptable. El costo de la atención hospitalaria mostró diferencia entre los grupos: menos de U.S. \$ 1,1 en 55\% de casos y $76 \%$ y controles $(\mathrm{OR}=0.46(0.19$ 0.84) $\mathrm{P}=0.007$ ), lo cual es explicable porque los primeros requirieron más exámenes, medicamentos y hospitalización que los otros. Es claro que estos pacientes tienen muy bajos ingresos económicos mensuales (menos de U.S. \$ 111 en 73\% de ellos) y que el dinero disponible para invertir en la consulta es bajo (menos de U.S. \$ 1,1 en $56 \%$ ). Un hecho que elimina muchas de las diferencias en materia económica entre ambos grupos es que están afiliados al sistema de seguridad social en salud en proporción similar ( $64 \%$ casos, $66 \%$ controles, $\mathrm{OR}=1.09$ (0.58-2.02 $\mathrm{P}=0.796)$.

Un análisis posterior de los datos mediante un modelo multivariado permitirá el análisis y control de efectos confusores potenciales y evaluar las posibles interacciones entre las variables estudiadas, aspectos que podrían modificar los resultados del análisis univariado aquí presentado. 


\section{Conclusiones}

El haber tenido antecedente malaria falciparum durante el último año parece haber protegido a algunos pacientes de que la infección actual por falciparum se complicase. Encontramos el doble de probabilidad de complicarse en los pacientes que hicieron uso de medicamentos antimaláricos antes de consultar; en su mayoría los pacientes no emplearon correctamente los antimaláricos ya que tomaron el medicamento inadecuado para tratar la malaria falciparum, o tomaron subdosis de la droga. El mal uso de antimaláricos podría llevar a una selección de parásitos, favoreciendo una recrudescencia de la parasitemia que lleva a un mayor deterioro clínico, o quedando un parásito más virulento en el paciente (asunto que no pudo establecerse en este estudio).

Aunque la metodología de este trabajo no permite establecer el valor pronóstico de los signos de peligro para desarrollar $\mathrm{MC}$, encontramos 3 veces más estos signos en los pacientes que hicieron complicaciones. Debe profundizarse en el estudio del valor predictivo de los signos de peligro ya que algunos de estos signos como la coluria, frecuencia respiratoria alta e hiperpirexia, los encontramos en pacientes no complicados, sin embrago en otras regiones del mundo son usados para reconocer pacientes complicados o en riesgo de complicarse. El reconocimiento estos signos podría incorporarse en los programas de diagnóstico y tratamiento de la malaria, permitiendo la remisión oportuna de pacientes que están en riesgo de complicarse hasta un servicio médico y el inicio de un tratamiento adecuado.

El estudio permitió reconocer problemas serios como el suministro incorrecto de antimaláricos por el personal de salud y la automedicación, demora para consultar igual o superior a una semana en el $30 \%$ de los pacientes y demora para recibir atención efectiva en los centros de salud superior a 5 horas en el $93 \%$ de los pacientes.

Los factores que propician la presentación de MC en poblaciones semiinmunes pueden actuar de manera diferente en zonas de endemicidad alta, por tanto debe continuarse en el estudio de las complicaciones maláricas en regiones de transmisión baja o inestable y, de manera similar, debe profundizarse en el estudio de criterios diagnósticos diferentes a los propuestos por la OMS, los cuales parecen ser útiles especialmente en zonas de alta endemicidad.

\section{Declaración}

Los autores declaramos que la ejecución de este trabajo y la publicación de sus resultados carecen de conflicto de intereses.

\section{Referencias}

1. World Health Organization, UNICEF. World Malaria Report 2005. Global Malaria Situation, Malaria Burden p.11. Geneva, Switzerland 2005, pp.294.

2. Ariey F, Hommel D, Le Scanf C, Duchemin JB, Peneau C, Hulin A et al. Association of severe malaria with a specific Plasmodium falciparum genotype in French Guiana. $J$ Infec Dis 2001; 184(15):237-241.

3. Greenwood B. The molecular epidemiology of malaria. Trop Med Int Health 2002; 7(12):1012-21.

4. Luxemburger C, Ricci F, Nosten F, Raimond D, Bathet S, White NJ. The epidemiology of severe malaria in an area of low transmission in Thailand. Trans R Soc Trop Med Hyg 1997; 9(3):256-62.
5. Imbert P, Sartelet I, Rogier C, Ka S, Baujat G, Candito D. Severe malaria among children in a low seasonal transmission area, Dakar, Senegal: influence of age on clinical presentation. Trans R Soc Trop Med Hyg 1997, 91 : $22-244$.

6. Banzal S, Ayoola EA, Sammani EE, Rahim SI. The clinical pattern and complications of severe malaria in the Gizan region of Saudi Arabia. Ann Saudi Med 1999; 19(4):19498.

7. Artavanis-Tsakonas K, Tongren JE and Riley EM. The war between the malaria parasite and the immune system: immunity, immunoregulation and immunopathology. Clin Exp Immunol 2002, 133:145-52. 
8. Friedman JF, Kwena AM, Mirel LB, Kariuki SK, Terlouw DJ, Phillips-Howard PA et al. Malaria and nutritional status among pre-school children: results from crosssectional surveys in western Kenya. Am J Trop Med Hyg 2005; 73(4):698-704.

9. Sarthou JL, Angel G, Aribot G, Rogier C, Dieye A, Balde AT et al. Pronostic value of anti-Plasmodium falciparum especific immunoglobulin G3, cytoquines and their soluble receptors in West African patients with severe malaria. Infect Immunol 1997; 65:3271-6.

10. Guthmann JP, Hall AJ, Jaffar S, Palacios A, Lines J, LlanosCuentas A. Environmental risk factors for clinical malaria: a case-control study in the Grau region of Perú. Trans $R$ Soc Trop Med Hyg 2001; 95:577-83.

11. Méndez F., Carrasquilla G, Muñoz A. Risk factors associated with malaria infection in an urban setting. Trans R Soc Trop Med 2000; 94(4):367-71.

12. Carme B, Plassart H, Senga P, Nzingoula S. Cerebral malaria in African children: socioeconomic risk factors in Brazzaville, Congo. Am J Trop med Hyg 1994; 50(2):131-6.

13. Sirima SB, Konaté A, Tiono AB, Convelbo N, Cousens S, Pagnoni F. Early treatment of childhood fevers with prepackaged antimalarial drugs in the home reduces severe malaria morbidity in Burkina Fasso. Trop Med Int Health 2003; 8(2):133-9.

14. Reilley B, Abeyasinghe R, Pakianathar MV. Barriers to prompt and effective treatment of malaria in northern Sri Lanka. Trop Med Int Health 2002; 7(9):744-9.

15. World Health Organization. Severe falciparum malaria. World Health Organization, Communicable Diseases Cluster. Trans R Soc Trop Med Hyg 2000; 94 (Suppl 1):S190 .

16. Gonzalez L, Guzmán M, Carmona J, Lopera T, Blair S. Características clinico-epidemiológicas de 291 pacientes hospitalizados por malaria en Medellín (Colombia). Acta Med Col 2000; 25: 163-70.

17. Castañeda AM, Daza C. Niveles de parasitemia por Plasmodium falciparum y algunos factores relacionados. Urabá y Bajo Cauca, Departamento de Antioquia, 19881989. Tesis de posgrado. Facultad Nacional de Salud Pública, Universidad de Antioquia, Medellín, 1989.

18. Allen SJ, O`Donell A, Alexander ND, Clegg JB. Severe malaria in children in Papua New Guinea. Q J M Monthly JAss Physicians 1996; 89(10):779-88.

19. Shankar A. Nutritional modulation of malaria morbidity and mortality. J Infect Dis 2000; 182 (suppl): S37-63.

20. Nacher M, Singhasivanon $P$, Treeprasertsuk $S$, Vannaphan S, Traore B, Looareesuwan S. Intestinal helminths and malnutrition are independently associated with protection from cerebral malaria in Thailand. Ann Trop Med Parasitol 2002; 96 (1): 5-13.
21. Nacher M, Singhasivanon P, Traore B, Vannaphan S, Gay $\mathrm{F}$, Chindanond D et al. Helminth infections are associated with protection from cerebral malaria and increased nitrogen derivatives concentrations in Thailand. Am J Trop Med Hyg 2002; 66 (3): 304-9.

22. Le Hesran JY, Akiana J, Ndiaye el HM, Dia M, Senghor P, Konate L Severe malaria attack is associated with high prevalence of Ascaris lumbricoides infection among children in rural Senegal. Trans R Soc Trop Med Hyg 2005; 99(2):161-3.

23. World Health Organization. Management of severe malaria: a practical handbook. $2^{\mathrm{a}}$ edition. Geneva, 2000.

24. Koram KA, Benett S, Adiamah JH, Greenwood BM. Socioeconomic determinants are not major risk factors for severe malaria in Gambian children. Trans R Soc Trop Med Hyg 1995, 89: 151-4.

25. Chaves SS; Rodrigues LC. An initial examination of the epidemiology of malaria in the State of Roraima, in the Brazilian Amazon Basin. Rev Inst Med Trop Säo Paulo 2000; 42(5):269-75

26. Velásquez VA. Estudio de casos y controles apareados de los factores de riesgo de la malaria que favorecen el contacto hombre-vecto en un area endémica de Plasmodium vivax, Venezuela. SITUA 1997; (9):28-34.

27. Vaca MA. Fatores de risco e perspectivas de controle da malária na fronteira de Chiapas, México, com Guatemala. Risk factors and perspectives of control of the malaria in the border of Chiapas, Mexico, with Guatemala. Rio de Janeiro; s.n; 1994. xii,142 p.

28. Martínez EF. Malária na gravidez: estudo de pacientes do Instituto de Medicina Tropical do Amazonas, Brasil, 19901997 / Malaria in the pregnancy: study of patients of the Instituto de Medicina Tropical do Amazonas, Brazil, 1990-1997. Rio de Janeiro; s.n; 1998. 142 p.

29. Almeida NJ, Araujo FJ, Kobal CR, Monteiro AI, Rodarte AR, Zicker F. Malária grave por P. falciparum. Avaliaçäo das falhas no diagnóstico e na conduta terapêutica em pacientes antes de sua internaçäo em Hospital de Referência. Rev Patol Trop 1995; 24(1):1-9.

30. Silva FC, Carmo RA, Bastos SM. Estudo retrospectivo de 49 casos de malária internados no Hospital das Clínicas UFMG, de 1981 a 1992. Rev Med Minas Gerais 1995; 5(4):230-5.

31. Kirchgatter K, Portillo Hdel A. Association of severe noncerebral Plasmodium falciparum malaria in Brazil with expressed PfEMP1 DBL1 alpha sequences lacking cysteine residues. Mol Med 2002 Jan;8(1):16-23.

32. Colombia. Ministerio de Salud. Informe de enfermedades transmitidas por vectores. Informe quincenal epidemiológico nacional 2006;11(4):49-64.

33. Colombia. Ministerio de Salud. Informe de enfermedades transmitidas por vectores. Informe quincenal epidemiológico nacional. 2005; 10(3):39-43. 
34. Colombia, Ministerio de Salud. Guía de atención de la malaria. Bogotá, Diario Oficial Edición 43956, Marzo de 2000. p. 173-184.

35. World Health Organization. 1996. Malaria. A manual for community health workers. Geneva, Switzerland.

36. Lopez AF, Schmunis G. Diagnóstico de malaria. Washington, Organización Panamericana de la Salud (O.P.S.), 1988. Publicación Científica No. 512.

37. Montoya L, Maestre A, Carmona J, Lopes D, Do Rosario V, Blair S. Plasmodium falciparum: diversity studies of isolates from two Colombian regions with different endemicity. Exp Parasitol 2003; 104:14-19.

38. Echeverri M, Tobón A, Alvarez G, Carmona FJ, Blair S. Clinical and laboratory findings of Plasmodium vivax malaria in Colombia, 2001. Rev Inst Med Trop Sao Paulo 2003. 45(1):29-34.

39. Piñeros JG, Padilla JC, Montoya R, García I. Malaria grave en un hospital de segundo nivel del Pacifico colombiano, 2001. Informe Epidemiológico Nacional Quincenal Colombia 2001; 6(24):361-6.

40. Braunwald E, Fauci AS, Kasper DL, Hauser SL, Longo DL, Jameson JL (editores). Harrison's 2001. Principles of internal medicine. 15 ${ }^{\text {a }}$ Edición. Nueva York: Mac GrawHill, 2001
41. Emergency Medicine Bulletin Board System. Diagnosis of acute renal failure. En: www. Embbs.com/cr/ rf)rf3.html. Consultado febrero 15 de 2004.

42. Kun JF, Schmidt-Ott RJ, Lehman LG, Lell B, Luckner D, Greve B, Matousek P et al. Merozoite surface antigen 1 and 2 genotypes and rosetting of Plasmodium falciparum in severe and mild malaria in Lambarene, Gabon. Trans $R$ Soc Trop Med Hyg 1998; 92(1):110-4

43. Ranjit MR, Das A, Das BP, Das BN, Dash BP, Chhotray GP. Distribution of Plasmodium falciparum genotypes in clinically mild and severe malaria cases in Orissa, India. Trans R Soc Trop Med Hyg 2005; 99(5):389-95.

44. Amodu OK, Adeyemo AA, Ayoola OO, Gbadegesin RA, Orimadegun AE, Akinsola AK et al. Genetic diversity of the msp-1 locus and symptomatic malaria in south-west Nigeria. Acta Trop 2005; 95(3):226-32.

45. Robert, F, Ntoumi, F, Angel G, Gandito D, Rogier D, Fandeur T et al. Extensive genetic diversity of Plasmodium falciparum isolates collected from patients with severe malaria in Dakar, Senegal. Trans R Soc Trop Med Hyg 1996; 90:704-11.

recebido em: 12/04/06 versão final reapresentada em: 04/08/06 aprovado em: 09/08/06 